\title{
EFEITO DO ALBEDO EM CÂNIONS URBANOS DE CIDADES MÉDIAS BRASILEIRAS
}

\author{
GIORDANO, Dominique Elena (1); ERELL, Evyatar (2); KRÜGER, Eduardo (3) \\ (1) UTFPR/IFPR, dominique.giordano@ifpr.edu.br (2) Ben Gurion University, erell@bgu.ac.il, (3) \\ UTFPR, ekruger@utfpr.edu.br
}

\begin{abstract}
RESUMO
Albedo é uma propriedade dos materiais que indica sua capacidade de refletir a radiação solar. Quando exposto ao sol, materiais de alto albedo refletirão mais a radiação solar incidente, e portanto, mais baixas suas temperaturas superficiais, razão pela qual são chamados "materiais frescos". O uso destes materiais em superfícies urbanas tem sido elencado em diversos estudos como possível estratégia para a mitigação dos efeitos da Ilha de Calor Urbano, especialmente em climas quentes, por reduzirem as temperaturas superficiais e consequentemente, a temperatura do ar. Este estudo objetivou avaliar os potenciais efeitos do uso de materiais de alto albedo em um cânion urbano, para cidades brasileiras de médio porte. A pesquisa visou verificar se o incentivo ao uso de maiores albedos poderia ser adotado no rol de políticas públicas, a fim de orientar o crescimento de cidades para uma melhor qualidade ambiental. $\mathrm{O}$ efeito do albedo sobre a temperatura do ar de um cânion hipotético foi simulado com um modelo matemático de micro escala, o Canyon Air Temperature (CAT), e o conforto do pedestre, através do Índice de Estresse Térmico (ITS), no centro do cânion. Ambos os efeitos foram simulados para um dia de céu claro de verão e outro de inverno, em oito diferentes cidades de semelhante tamanho populacional, cada uma localizada em uma Zona Bioclimática brasileira. Os resultados mostram que elevar o albedo de uma rua, isoladamente, tem efeito pouco significativo sobre a temperatura do ar, embora impacte mais fortemente o ITS.
\end{abstract}

Palavras-chave: Albedo, Conforto Térmico, Cânion Urbano, Pedestres, Planejamento Urbano.

\begin{abstract}
Albedo is a material property that refers to its ability to reflect solar radiation. When exposed to sun, a high albedo material will have a higher reflection of incident sunlight, and therefore, lower will be its surface temperature, reason why such materials are called "cool materials". The use of these materials over urban surfaces has been highlighted in several studies as a possible strategy for mitigating Urban Heat Island effects, mainly in hot climates, for reducing surface temperatures, and consequently, air temperature. This study aimed to evaluate potential effects of locally adopting high albedo materials over an urban canyon in medium-sized Brazilian cities. The research purpose was to verify if higher albedo materials would positively contribute to planning initiatives, in order to guide the growth process of cities towards a better urban environment. The albedo effect on air temperature inside the canyon was simulated using a micro scale mathematical model, the Canyon Air Temperature (CAT); the derived pedestrian comfort was assessed, by means of the Index of Thermal Stress (ITS), calculated for a person standing in the middle of the canyon. Both effects were simulated for a clear day in winter and in summer, for eight different cities of approximately same population size, each one in a different Bioclimatic Zone of Brazil. Results shows that raising albedo of one single street has a minor effect on air temperature, while yielding a stronger impact on ITS.
\end{abstract}

Keywords: Albedo, Thermal Comfort, Street Canyon, Pedestrian, Urban Planning. 


\section{INTRODUÇÃO}

Nos últimos anos, considerável esforço de pesquisa foi voltado para o fenômeno das Ilhas de Calor Urbano (ICU), o qual é caracterizado pelo aumento da temperatura superficial e do ar sobre áreas urbanas em comparação ao seu entorno rural. A popularidade do tema pode estar associada à estreita relação entre algumas das consequências da ICU e o tema da sustentabilidade, como por exemplo, aumento do consumo de energia (RADHI; SHARPLES, 2013), concentração de poluentes (AGARWAL; TANDON, 2010), mudanças nos padrões climáticos locais (HOFFMANN; SCHLUENZEN, 2013) e conforto térmico (RODRIGUES DA SILVA et al, 2010).

Dentre as estratégias para a mitigação dos efeitos da ICU, muitos estudos citam o uso extensivo de materiais de alto albedo não apenas em coberturas (JACOBSON; TEN HOEVE, 2012), mas também em pavimentos (CARNIELO; ZINZI, 2013).

Emmanuel e Fernand (2007) sugerem que a temperatura do ar, isoladamente, não é um bom parâmetro para avaliar os benefícios de estratégias de mitigação da ICU, e que ao invés disso, deve ser usada de forma combinada com índices de conforto humano. Alguns estudos têm demonstrado que embora o aumento do albedo possa reduzir as temperaturas de superfície e do ar, a maior quantidade de radiação refletida incidirá sobre pedestres afetando seu conforto térmico (ERELL et al, 2013), e sobre janelas, aumentando a carga térmica nestes ambientes, podendo resultar em maior consumo de energia (YAGHOOBIAN; KLEISSL, 2012).

O objetivo deste trabalho foi explorar os efeitos do uso de materiais de alto albedo nas cidades médias brasileiras. A hipótese inicial é a de que para a maior parte do território, situado em clima tropical, embora o aumento do albedo possa reduzir a temperatura do ar, pode haver um aumento no desconforto experimentado ao nível do pedestre. Nas regiões de clima mais frio, o efeito do albedo poderia melhorar o conforto de pedestres no inverno, ainda que o piore no verão. Conhecer esses efeitos é útil para identificar se essa seria uma prática a ser encorajada ou não nas cidades brasileiras, por meio de políticas públicas.

\section{MÉTODO}

\subsection{ESCOLHA DOS CENÁRIOS DE SIMULAÇÃO}

O resultado da alteração do albedo pode ser afetado pelas condições locais, como clima e geometria urbana. Para garantir a diversidade de cenários, foi selecionada uma cidade de cada uma das 8 Zonas Bioclimáticas brasileiras (ZB), definidas na Norma Brasileira de Desempenho Térmico (NBR 15220:2005). Buscaram-se cidades de porte médio, com tamanho populacional o mais semelhante possível (Tabela 1).

Para este estudo exploratório, somente um dia de verão e um de inverno foram simulados usando o Ano Meteorológico Típico (TMY) de cada cidade. O foco da análise foi restrito ao período diurno. Devido à falta de informações de nebulosidade, a curva diária de radiação solar direta foi observada para assegurar condição de céu claro, escolhendo entre os cinco dias com a Máxima Diária de Radiação Global com valor mais próximo de $95 \%$ da Máxima Mensal. As ZBs 2 e 7 seguiram regra diferenciada devido aos seus altos níveis de radiação difusa: foi escolhido o dia de menor porcentagem de radiação difusa relativamente à global (Figura 1). Ainda, para a ZB7, considerando sua proximidade ao Equador, o período considerado como mais crítico foi próximo ao equinócio (outubro), quando ocorrem as máximas elevações solares. 
Tabela 1 - Cidades escolhidas para o estudo

\begin{tabular}{|c|c|c|c|c|c|c|c|c|}
\hline ZB & Pop. (milhões) & UF & Cidade & Lat. & Long. & Grupo & Dia Ver. & Dia Inv. \\
\hline 1 & 0,357 & PR & Maringá & $-23,41$ & $-51,93$ & b & $01 / 12$ & $13 / 06$ \\
\hline 2 & 0,125 & RS & Uruguaiana & $-29,84$ & $-57,08$ & a & $06 / 12$ & $22 / 06$ \\
\hline 3 & 0,516 & MG & Juiz de Fora & $-21,77$ & $-43,36$ & c & $10 / 12$ & $21 / 06$ \\
\hline 4 & 0,318 & SP & Franca & $-20,58$ & $-47,38$ & d & $01 / 12$ & $13 / 06$ \\
\hline 5 & 0,463 & RJ & Campos & $-21,72$ & $-41,34$ & c & $22 / 12$ & $23 / 06$ \\
\hline 6 & 0,207 & SP & Pres. Prudente & $-22,12$ & $-51,40$ & c & $25 / 12$ & $08 / 06$ \\
\hline 7 & 0,247 & MA & Imperatriz & $-5,56$ & $-47,46$ & e & $04 / 10$ & $21 / 06$ \\
\hline 8 & 0,327 & ES & Vitória & $-20,32$ & $-40,32$ & d & $13 / 12$ & $08 / 06$ \\
\hline
\end{tabular}

Fontes: [População] Instituto Brasileiro de Geografia e Estatística - IBGE (2010);

[Coordenadas Estações Meteorológicas] Arquivos TMY, ajustadas com Google Earth (2014).

Figura 1 - Distribuição da Radiação Solar Direta e Difusa (TMY)

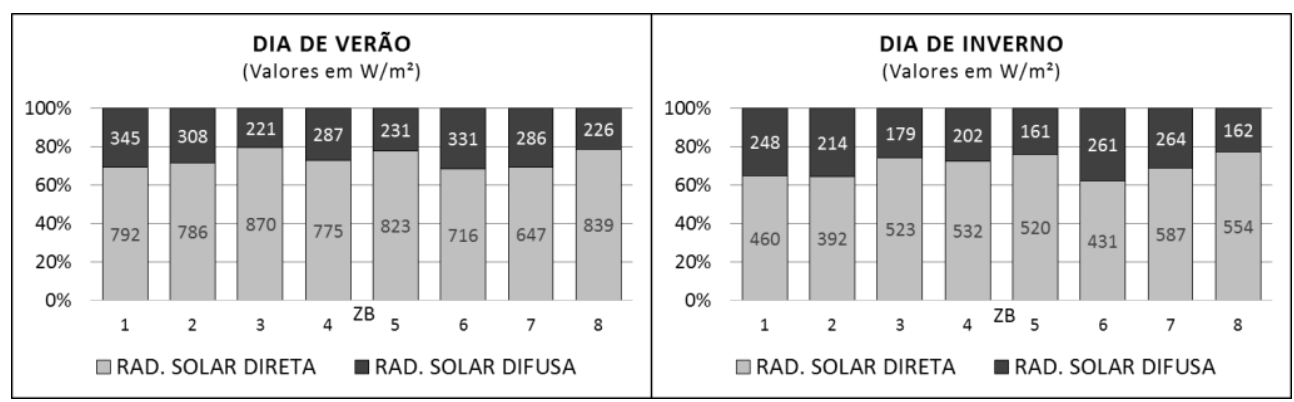

A geometria do cânion representa edifícios com a altura máxima permitida sem o uso de elevador (térreo e 4 andares), com cerca de $15 \mathrm{~m}$ de altura, separados por via local, resultando em cânion de razão altura/largura $(\mathrm{H} / \mathrm{W})$ de 1 . Esta configuração é plausível como futuro adensamento de bairros residenciais em cidades médias. Trabalhou-se com as orientações do eixo do cânion Norte-Sul (N-S) e Leste-Oeste (E-W). Para os cenários de albedo baixo $(\mathrm{AB})$, os valores do coeficiente de reflexão solar foram considerados para pavimento de asfalto $(0,15)$ e paredes escuras $(0,2)$; e, de albedo alto (AA), pavimento em concreto $(0,45)$ e paredes claras $(0,7)$.

\subsection{SIMULAÇÕES}

As simulações, realizadas com o modelo matemático de micro escala Canyon Air Temperature (CAT) (ERELL; WILLIAMSON, 2006), geraram como resultados para o interior do cânion valores horários das seguintes variáveis: temperatura do ar, umidade relativa, radiação solar, temperatura das superfícies e velocidade do ar ao nível do pedestre. Os principais dados de entrada são: geometria e orientação do cânion, albedo de pavimento e paredes, dados de uma Estação Meteorológica (WS) próxima, e fontes de umidade num raio de $1 \mathrm{~km}$ ao redor da WS e do cânion simulado (CS) (Figura 2). O CAT realiza a transposição do balanço de energia e perfis de vento da WS para as condições urbanas. Baseado no perfil logarítmico de velocidade do vento, usa-se o comprimento de rugosidade $\left(z_{0}\right)$ e o deslocamento do plano zero $(d)$ na WS para calcular a velocidade de fricção e com ela, a velocidade do vento geostrófico $\left(U_{g}\right)$, ou seja, no topo da Camada Limite Atmosférica. Assumindo $U_{g}$ igual sobre WS e CS, as condições do entorno urbano $(z 0 ; d)$ permitem então o cálculo inverso, obtendo o perfil de vento sobre o CS, e a 
velocidade no nível da cobertura (ERELL; PEARLMUTTER; WILLIAMSON, 2011). Para a velocidade do vento no nível do pedestre, usa-se a correlação de MacDonald (MACDONALD, 2000).

\section{Figura 2 - Exemplo dos 33 Setores usados para cálculo da disponibilidade de umidade no entorno do CS e da WS, sobre imagens Landsat8}

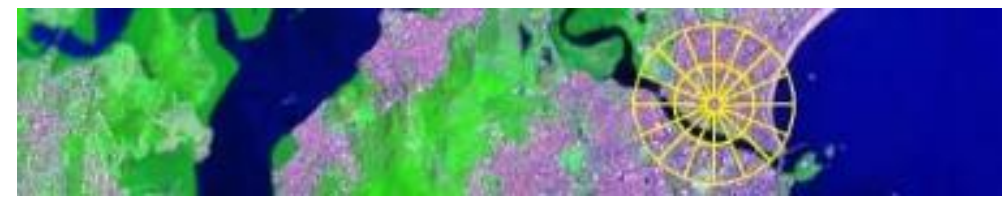

A disponibilidade de umidade permite ao CAT determinar o calor sensível e latente no balanço de energia, baseado no trabalho de Grimmond e Oke (2002). A umidade disponível é estimada da porcentagem de corpos d'água e áreas vegetadas no raio de $1 \mathrm{~km}$, dividido em 33 setores ao redor da WS e CS (Figura 2). Pequena imprecisão na localização da WS pode alterar a disponibilidade de umidade, por isso a seleção das cidades excluiu aquelas onde não havia confirmação visual da posição da WS em imagens aéreas. As fontes de umidade nos setores foram calculadas no SPRING 5.2.6 (CAMARA et al, 1996), usando imagens Landsat8, que foram classificadas pelo método da Máxima Verossimilhança, aplicado sobre as bandas infravermelhas 7 e 5 (identificação da umidade no solo e vegetação) e a banda 2, azul (distinção entre solo e vegetação). Para melhorar o cálculo da área, refinou-se a resolução do grid de 30 para $15 \mathrm{~m}$, fusionando as bandas de trabalho com a banda 8, pancromática, com o Spectral Transformer for Landsat8 Imagery. A reprojeção para o hemisfério sul, sistema SIRGAS 2000 usou QGIS 2.2.0 Valmiera. Todos os softwares e imagens utilizados são de acesso gratuito.

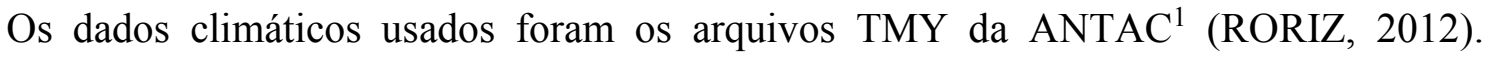
Infelizmente, não os arquivos não contém dados de precipitação e nebulosidade, e a base de dados online do INMET ${ }^{2}$ não fornece dados horários destas variáveis. No entanto, suas influências estão implícitas nos valores de umidade relativa e radiação solar, respectivamente. A nebulosidade não foi necessária para que o CAT estimasse a Radiação Infravermelha da abóbada pois esses valores estavam disponíveis no TMY.

\subsection{AVALIAÇÃO DO CONFORTO TÉRMICO}

A avaliação do conforto térmico do pedestre usou o Índice de Estresse Térmico (ITS). Desenvolvido por Givoni (1963), e adaptado para ambientes externos por Pearlmutter, Berliner e Shaviv (2007), representa a taxa de calor a ser transferida ao ambiente por transpiração (calor latente) para que seja mantido o equilíbrio térmico do corpo (Equação 1). Baseia-se somente nas trocas de energia entre corpo e ambiente, sem conversão a uma temperatura equivalente. A Tabela 2 mostra a correlação entre ITS e níveis de conforto.

$$
\text { ITS }=\left(R_{n}+C+M-W\right) / f
$$

onde, $\mathrm{R}_{\mathrm{n}}=$ radiação líquida, sobre cilindro de área equivalente ao do corpo, em W/m²;

$\mathrm{C}=$ perda de calor sensível por convecção, em W/m²;

$\mathrm{M}$ = calor gerado devido ao metabolismo basal, em W/m²;

$\mathrm{W}$ = calor gerado devido ao trabalho (atividade física), em W/m²;

$\mathrm{f}=$ coeficiente de eficiência da transpiração.

\footnotetext{
${ }^{1}$ ANTAC: Associação Nacional de Tecnologia do Ambiente Construído.

2 INMET: Instituto Nacional de Meteorologia.
} 
Tabela 2 - Níveis de Conforto do Pedestre pelo ITS

\begin{tabular}{|c|c|c|c|c|}
\hline ITS & & & +480 & $\left(\mathrm{~W} / \mathrm{m}^{2}\right)$ \\
\hline 3- FRIO & 4-CONFORTAVEL & 5-MORNO & 6- QUENTE & 7- MUITO QUENTE \\
\hline
\end{tabular}

\section{RESULTADOS E DISCUSSÕES}

\subsection{CONDIÇÕES CLIMÁTICAS NO INTERIOR DO CÂNION}

Figura 3 - Efeito do albedo sobre a temperatura do ar no interior do cânion (ZB3)

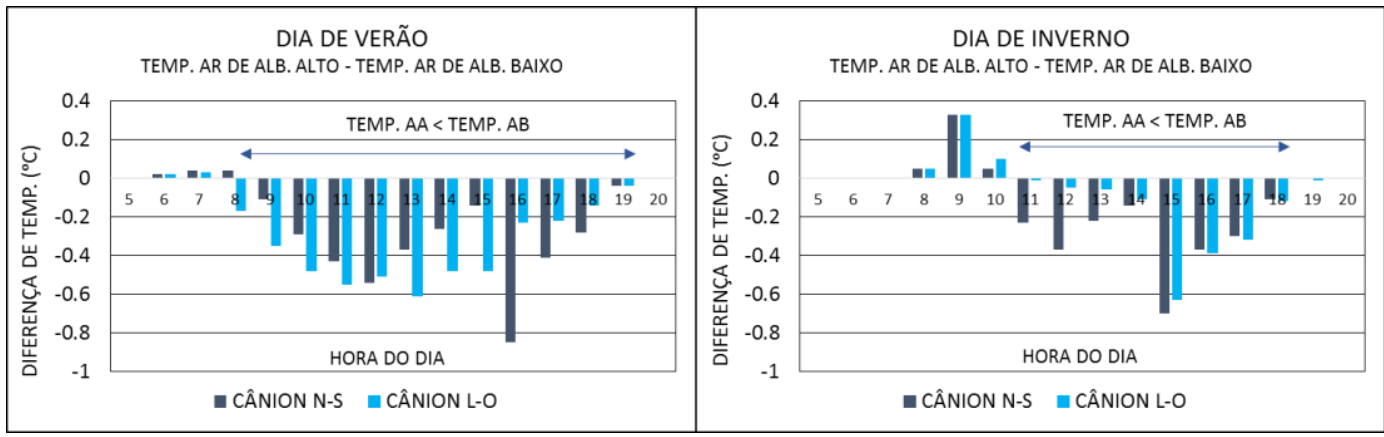

Figura 4 - Panorama da influência do albedo sobre as temperaturas do ar

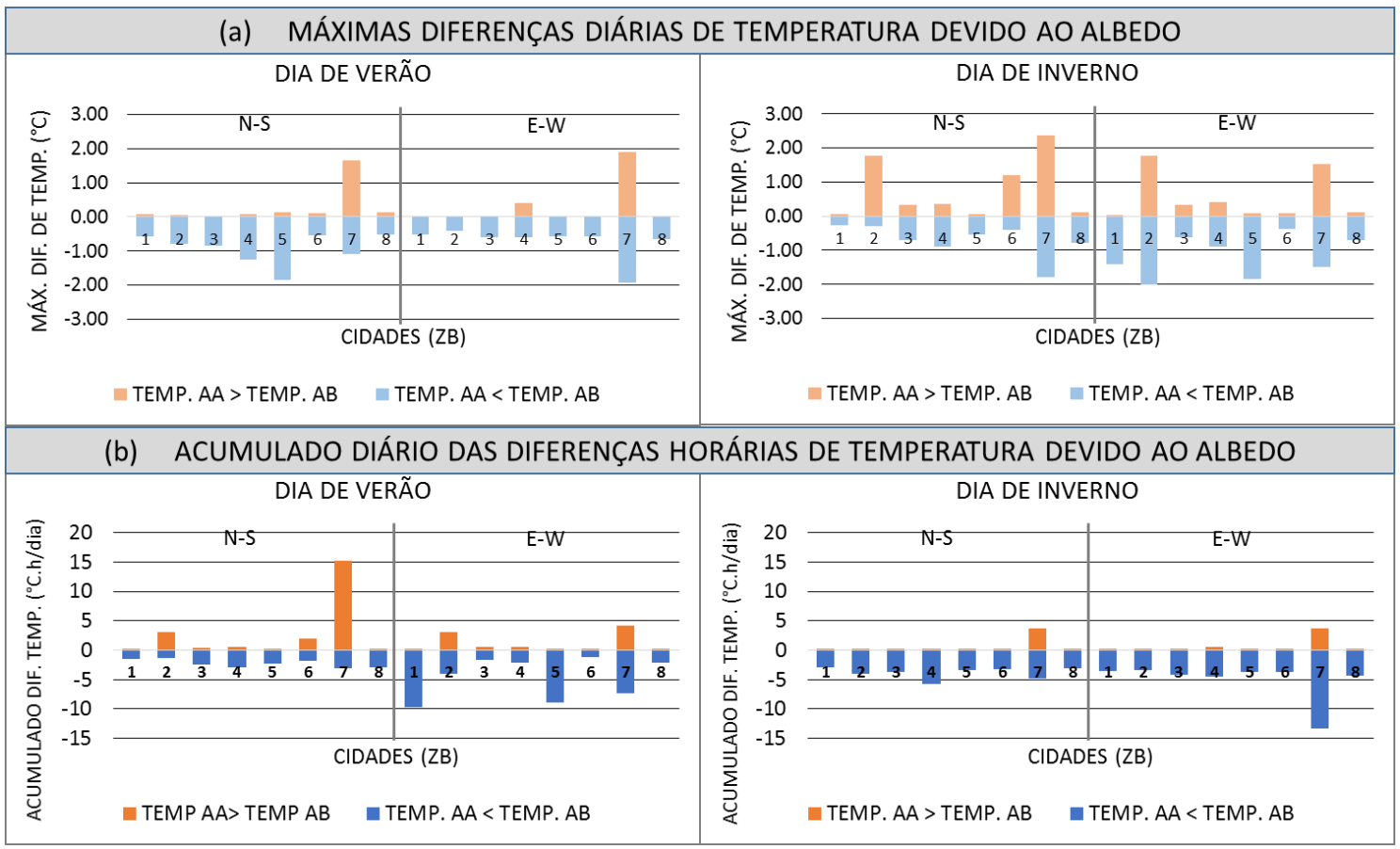

Comparando os cenários de albedo alto (AA) e albedo baixo ( $\mathrm{AB}$ ), há tendência de temperaturas de ar mais baixas com $\mathrm{AA}$, da ordem de $0,5^{\circ} \mathrm{C}$. A Figura 3 mostra para a cidade da ZB3, a distribuição das diferenças horárias de temperatura devido ao albedo $\left(\Delta \mathrm{T}=\mathrm{T}_{\mathrm{AA}}-\mathrm{T}_{\mathrm{AB}}\right)$, enquanto a Figura 4-a apresenta, para todas as cidades analisadas, os valores extremos de $\Delta \mathrm{T}$ para o dia analisado. Em ambas, $\Delta \mathrm{T}>0$ indica temperatura mais alta em AA, e $\Delta \mathrm{T}<0$, temperatura mais baixa em AA, comparadas ao AB. A Figura 4-b mostra o acumulado diário das diferenças horárias devido ao albedo $\left(\Sigma_{\Delta \mathrm{T}}\right)$. Um valor 
extremo alto combinado com valor acumulado baixo, indica que a maior diferença de temperatura devido ao albedo é pontual no dia (ex.: ZB5/verão/N-S). O oposto, valor extremo baixo e acumulado alto, indica que ainda que a diferença de temperatura devido ao albedo seja pequena, ela é recorrente, e se estende por mais tempo no dia (ex.: ZB5/verão/L-O). Analisando a Figura 4-b nota-se na situação de inverno, apenas a cidade 7/L-O se destaca com temperaturas do ar menores devido ao AA. Na situação de verão, apenas a orientação L-O mostra diferenças significativas para as cidades 1,5 e 7 .

Os cenários previstos indicam que, na micro-escala (uma rua isoladamente), há uma contribuição do alto albedo na redução da temperatura do ar, mas ela é em geral, de pequena magnitude, o que pode ser explicado em parte pela absorção da radiação refletida pelas superfícies adjacentes, cancelando em parte o efeito inicial da alta refletância, e em parte pelo efeito da convecção que promove a mistura do ar no interior do cânion e com o ar acima do dossel urbano, diluindo o impacto local do albedo.

\subsection{EFEITO NAS TROCAS RADIANTES SOBRE PEDESTRES}

A magnitude do aumento de calor radiante sobre o pedestre varia bastante entre as cidades e cenários. A Figura 5 mostra os componentes do ITS para a cidade com máxima influência do albedo (ZB1). Os componentes de Radiação Solar Global (Global-SW) e Radiação Infra-Vermelha da abóbada (IR Celeste-LW) provém diretamente do céu e não são alterados pelo albedo, mantendo o mesmo valor em ambos os cenários (AA e AB).

Figura 5 - Componentes do fluxo de calor radiante sobre pedestres, comparando cenários de albedo baixo e alto

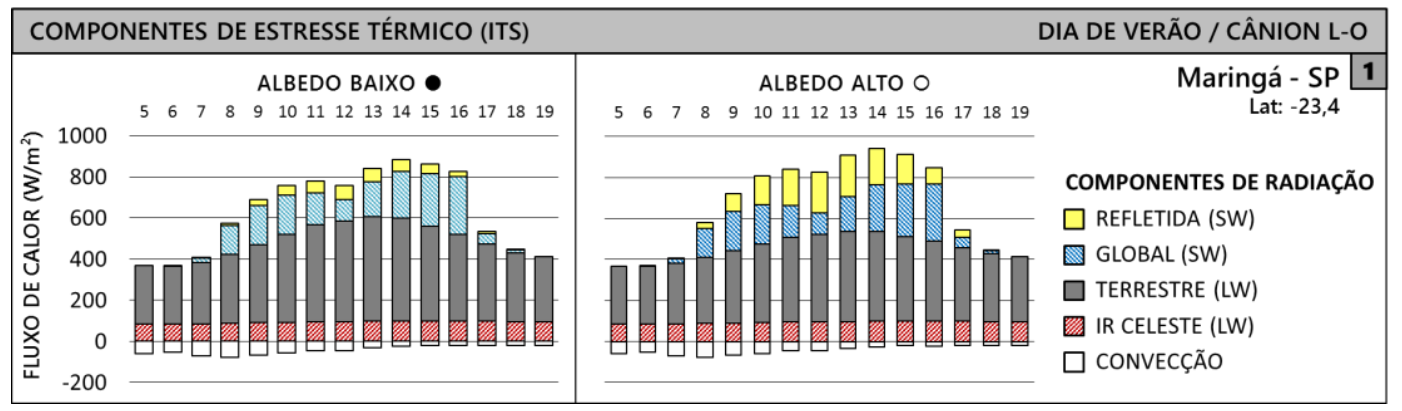

Proveniente do entorno, há os componentes de Radiação Solar Refletida (Refletida-SW) e Radiação Terrestre (Terrestre-LW). O primeiro, é radiação do tipo Onda Curta (SW) e é diretamente influenciado pelo albedo, aumentando com ele. O segundo, é do tipo Onda Longa (LW), emitida por qualquer superfície como resultado de sua temperatura, e se reduz com o aumento do albedo. No entanto, mudanças na temperatura ocorrem gradual e cumulativamente no tempo, enquanto a reflexão é um efeito instantâneo. Dentro do cânion, parte do efeito do albedo é cancelado, pois as superfícies adjacentes absorvem a radiação refletida pelas demais, aprisionando parte do calor, ao contrário das coberturas onde a radiação é refletida em direção à atmosfera. Dentre os componentes do ITS, a única que representa perda de calor é a Convecção (calor sensível), cujos valores não são altos uma vez que a velocidade do vento geralmente é baixa dentro do cânion. Para manter a temperatura do corpo estável, o pedestre deve compensar a diferença entre ganhos e perdas através da transpiração (calor latente).

Os resultados de várias cidades foram similares e, para ilustrar melhor os resultados, elas foram agrupadas de acordo com sua proximidade em latitude (Grupos a-e). Na Figura 6, as barras mostram a diferença causada pela mudança de albedo em cada componente do 
ITS, ou seja, o quanto um componente é acrescido ou diminuído pela substituição do $\mathrm{AB}$ por AA. O efeito do albedo sobre a Convecção (barras brancas) é tão pequeno que praticamente desaparece no gráfico. O saldo líquido dessas diferenças (linhas) representa o incremento de Estresse Térmico devido ao albedo.

Figura 6 - Diferença no fluxo radiante entre os cenários de alto e baixo albedo

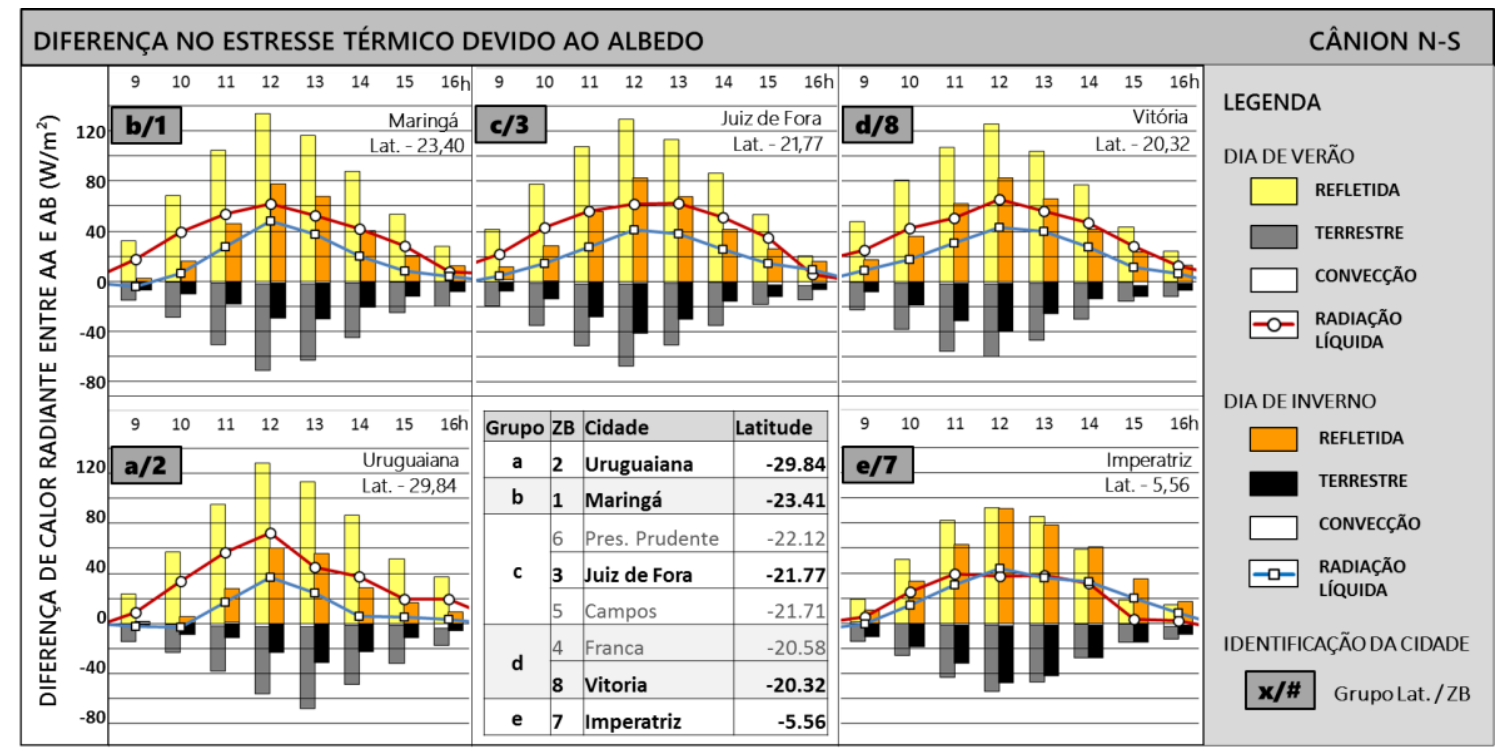

Quando o albedo é elevado, há uma troca entre componentes, aumentando a radiação Refletida-SW (barras amarelas e laranja) e diminuindo a Terrestre-LW (barras cinzas e pretas). Como a redução das temperaturas superficiais é defasada no tempo, e menos intensa que o aumento da reflexão, aumentar o albedo resulta em saldo positivo de radiação sobre o pedestre (linhas), aumentando seu Estresse Térmico (ITS).

Figura 7 - Máximo Incremento de Calor Radiante ao pedestre devido ao albedo
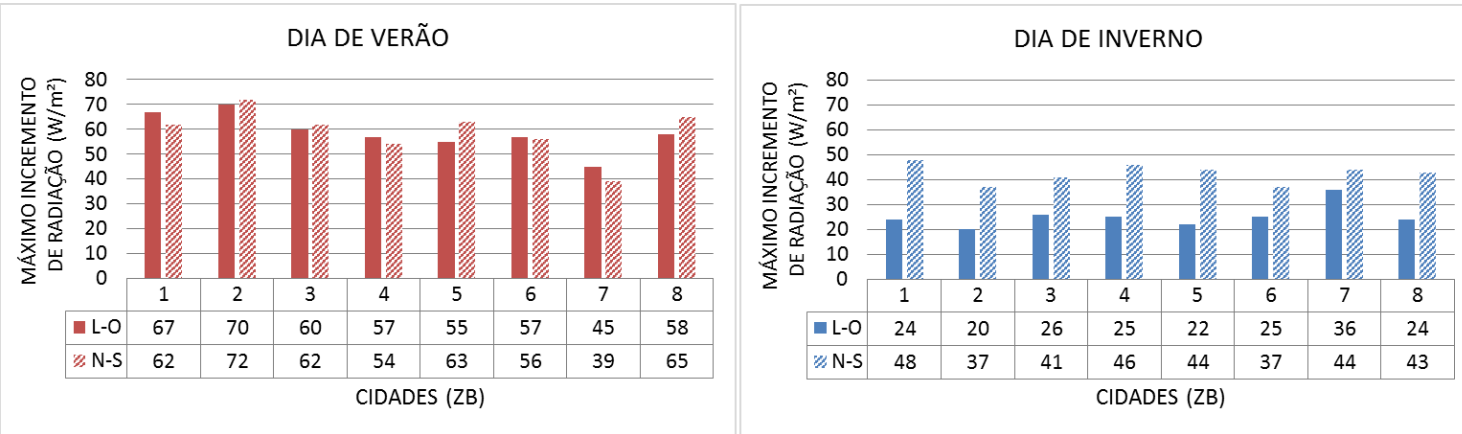

O incremento de Estresse Térmico é geralmente maior na condição de verão do que na de inverno, o que é consistente com maiores intensidades de radiação solar neste período. Por outro lado, os resultados sugerem menores incrementos para baixas latitudes, onde há menor variação sazonal na intensidade de radiação solar, e a elevação solar é mais alta, atingindo os pedestres principalmente sobre a cabeça e ombros, uma área de exposição corporal menor.

A magnitude do Máximo Incremento Diário de Calor Radiante para os dias estudados variou de 20 a $72 \mathrm{~W} / \mathrm{m}^{2}$, dependendo da estação e orientação do cânion (Figura 7). A média dos máximos de ambas as orientações variou de 29 a $40 \mathrm{~W} / \mathrm{m}^{2}$ na condição de inverno e de 42 a $71 \mathrm{~W} / \mathrm{m}^{2}$ na de verão. 


\subsection{SENSAÇÃo TÉRMICA DE PEDESTRES}

Devido ao sombreamento matutino e vespertino, os cânions N-S mostram um perfil típico de maiores fluxos radiantes concentrados em torno do meio dia, enquanto os L-W tem um perfil de valores altos mais distribuídos ao longo do dia, representando uma condição de maior estresse térmico para o pedestre (Figura 8). A intensidade desse efeito varia, é claro, de acordo com a latitude e estação do ano, mas é uma tendência geral.

$\mathrm{O}$ incremento de calor radiante devido ao albedo é pequeno se comparado ao total líquido de radiação sobre o pedestre. No entanto, observando os níveis de conforto térmico, notase que adicionar de 40 a $70 \mathrm{~W} / \mathrm{m}^{2}$ devido ao albedo nas horas mais quentes do dia representa um prejuízo considerável para o pedestre, especialmente ao se considerar que o benefício de menores temperaturas do ar é pequeno, não representando compensação significativa para o conforto térmico do pedestre.

\section{Figura 8 - Total Líquido de Estresse Térmico e Níveis de Conforto para ZB1}

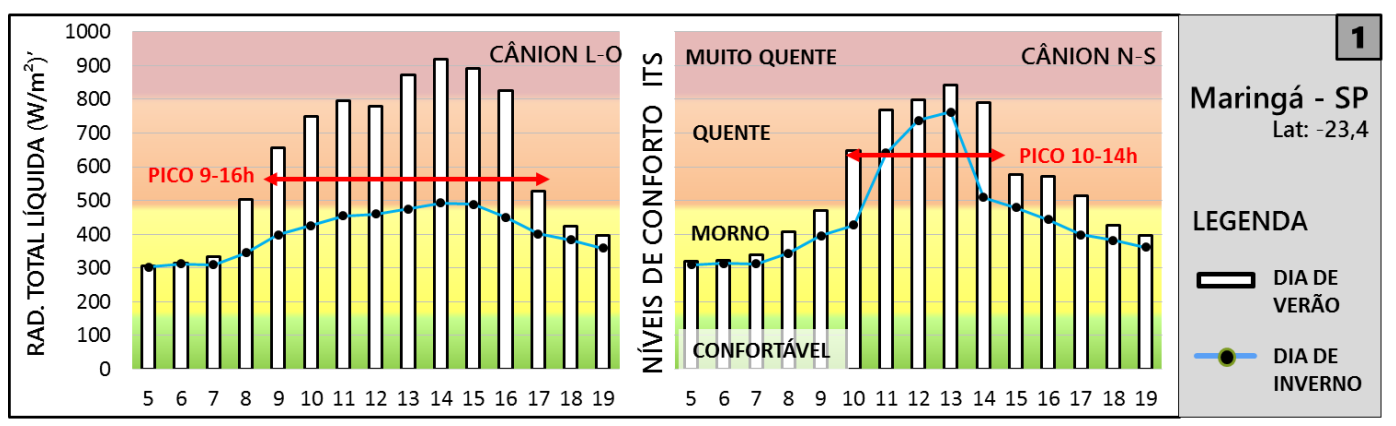

Figura 9 - Níveis de conforto do pedestre para todos os cenários

\begin{tabular}{|c|c|c|c|c|c|c|c|c|c|c|c|c|c|c|c|c|c|c|c|c|c|c|c|c|c|c|c|c|c|c|c|c|}
\hline \multirow{4}{*}{$\begin{array}{l}\stackrel{\mathbb{s}}{o} \\
\text { 오 } \\
\end{array}$} & \multicolumn{32}{|c|}{ ITS - Nível de Conforto do Pedestre (DIA DE VERÃO) } \\
\hline & \multicolumn{4}{|c|}{ ZB 1 } & \multicolumn{4}{|c|}{ ZB 2} & \multicolumn{4}{|c|}{$\overline{\mathrm{ZB} 3}$} & \multicolumn{4}{|c|}{$\overline{\mathrm{ZB} 4}$} & \multicolumn{4}{|c|}{ ZB 5} & \multicolumn{4}{|c|}{ ZB 6} & \multicolumn{4}{|c|}{ ZB 7} & \multicolumn{4}{|c|}{ ZB 8} \\
\hline & \multicolumn{3}{|c|}{$\leftrightarrow$} & & \multicolumn{2}{|c|}{$\leftrightarrow$} & & \multicolumn{2}{|c|}{$\leftrightarrow$} & & $\leftarrow$ & & & & $\leftarrow$ & & & & $\leftarrow$ & & & & $\leftarrow$ & & & & $\stackrel{\leftrightarrow}{\leftrightarrow}$ & & & \\
\hline & $\bullet$ & 0 & $\bullet$ & of & & o & 9 & 인 & 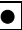 & 0 & $\bullet$ & 0 & $\bullet$ & & $\bullet$ & & $\bullet$ & & - & 의 & $\bullet$ & & $\bullet$ & 의 & $\bullet$ & 운 & $\bullet$ & 운 & & 인 & & 의 \\
\hline & 5 & 5 & 5 & 5 & 5 & 5 & & & & 5 & 5 & 5 & 5 & \begin{tabular}{l|l}
5 \\
\end{tabular} & \begin{tabular}{|l|}
5 \\
\end{tabular} & \begin{tabular}{l|l}
5 \\
\end{tabular} & 5 & 5 & 5 & 5 & 5 & 5 & 5 & 5 & 5 & 5 & 5 & 5 & 5 & 5 & 5 & 5 \\
\hline & 5 & 5 & 5 & 5 & 5 & 5 & & & & 5 & 5 & 5 & 5 & 5 & 5 & 5 & 5 & 5 & 5 & 5 & 5 & 5 & 5 & 5 & 5 & 5 & 5 & 5 & 5 & 5 & 5 & 5 \\
\hline 8 & 6 & 6 & 5 & 5 & 5 & 5 & & & & 6 & 5 & 5 & 6 & 6 & 5 & 5 & 6 & 6 & 5 & 5 & 5 & 5 & 5 & 5 & 6 & 6 & 5 & 5 & 6 & 0 & 6 & 6 \\
\hline 9 & 6 & 6 & 5 & 5 & 6 & $\epsilon$ & & & & & & & 6 & 6 & 5 & 5 & 6 & 7 & 6 & 6 & 6 & 6 & 6 & 6 & 6 & 6 & 6 & 6 & 7 & 7 & 6 & \\
\hline 10 & 6 & 6 & 6 & 6 & 6 & $\epsilon$ & & & & 6 & & 6 & 6 & 6 & 6 & 6 & 7 & 7 & 6 & 6 & 6 & 6 & \begin{tabular}{|l|} 
\\
\end{tabular} & 6 & 6 & 6 & 6 & 6 & 7 & & 6 & \\
\hline 11 & 6 & 6 & 6 & 6 & 6 & 6 & & & & 6 & 6 & 6 & 6 & 6 & 6 & 6 & 7 & 7 & 6 & 7 & 6 & 7 & 6 & 6 & 6 & 7 & 6 & 6 & 7 & & 7 & 7 \\
\hline 12 & 6 & 6 & 6 & 6 & 6 & 6 & 6 & 6 & 6 & 6 & 6 & 6 & 6 & 6 & 6 & 6 & 6 & 7 & 6 & 7 & 6 & 6 & 6 & 6 & 6 & 6 & \begin{tabular}{|l|}
6 \\
\end{tabular} & 6 & 7 & 7 & 6 & 7 \\
\hline 13 & 7 & 7 & 6 & 7 & 6 & 7 & & & & 7 & & 6 & 6 & 7 & 6 & 7 & 7 & 7 & 6 & 7 & 6 & 6 & 6 & 6 & 6 & 7 & 6 & 7 & 7 & 7 & 0 & \\
\hline 14 & 7 & 7 & 6 & 6 & 7 & 7 & 6 & 7 & 7 & 7 & 6 & 6 & 6 & 6 & 6 & 6 & 6 & 7 & 6 & 6 & 7 & 7 & 6 & 7 & 7 & 7 & 6 & 6 & 7 & & 6 & 6 \\
\hline 15 & 7 & 7 & 6 & 6 & 7 & 7 & 6 & 6 & & 7 & 6 & 6 & 7 & 7 & 6 & 6 & 6 & 6 & 6 & 6 & 7 & 7 & 6 & 6 & 6 & 6 & \begin{tabular}{|l|l|} 
\\
\end{tabular} & 10 & 6 & 7 & 6 & \\
\hline 16 & 7 & 7 & 6 & 6 & 7 & 7 & 6 & & & b & 5 & 5 & 7 & 7 & 6 & 6 & 6 & 6 & 6 & 6 & 7 & 7 & 6 & 6 & 6 & 6 & 6 & 6 & 6 & 6 & 6 & \\
\hline 17 & 6 & 6 & 6 & 6 & 6 & 6 & & & & 5 & 5 & 5 & 5 & 6 & 5 & 6 & 5 & 5 & 5 & 5 & 6 & 6 & 6 & 6 & 6 & 6 & \begin{tabular}{|l|}
6 \\
\end{tabular} & 6 & 5 & & 6 & \\
\hline 18 & 5 & 5 & 5 & 5 & 5 & 5 & 5 & 5 & 5 & 5 & 5 & 5 & 5 & 5 & 3 & 5 & 5 & 5 & 5 & 5 & 5 & 5 & 5 & 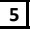 & 2 & 5 & 5 & & & & & \\
\hline TTES & & & & & & & & & & & & & -0 & test & & & & & & & & & & & & & & & & & & \\
\hline & & $\mathrm{Mu}$ & ito & que & ente & & & & Qu & ent & & & & & Mor & & & & & & & & nfor & rtáv & & & & & rio & & & \\
\hline & & & & & ITS & 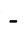 & $\Lambda$ & ve & 0 & $e$ & 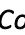 & & $r$ & to & do & $P$ & d & Hes & $s t r$ & $e$ & $|D|$ & $A$ & $D E$ & $E I I$ & $V V$ & $E R$ & VC & & & & & \\
\hline & & ZB & & & & $\overline{Z B}$ & & & & $\overline{Z B}$ & & & & ZB & & & & ZB & & & & ZB & 36 & & & ZB & & & & $\overline{Z B}$ & 8 & \\
\hline ఖి & $\leftarrow$ & & & 5 & $\leftarrow$ & & & & $\leftarrow$ & & & & $\leftarrow$ & & $\downarrow$ & & $\leftarrow$ & & $\uparrow$ & & $\leftarrow$ & & $\sqrt{3}$ & & $\leftarrow$ & & $\downarrow$ & & $\leftrightarrow$ & & $\uparrow$ & \\
\hline 두 & - & 0 & $\bullet$ & 이 & $\bullet$ & 으 & 의 & 의 & 인 & 의 & 부 & 의 & - & 인 & - & 이 & - & 0 & - & 으 & $\bullet$ & 0 & $\bullet$ & 0 & - & 이 & $\bullet$ & 으 & $\bullet$ & 으 & 으 & 의 \\
\hline 6 & 5 & 5 & 5 & 5 & 5 & 5 & 5 & 5 & 5 & 5 & 5 & 5 & 5 & 5 & 5 & 5 & 5 & 5 & 5 & 5 & 5 & 5 & 5 & 5 & 5 & 5 & \begin{tabular}{l|l|} 
\\
\end{tabular} & 5 & 5 & 5 & 5 & 5 \\
\hline 7 & 5 & 5 & 5 & 5 & 5 & 5 & 5 & 5 & 5 & 5 & 5 & 5 & 5 & 5 & 5 & 5 & 5 & 5 & 5 & 5 & 5 & 5 & 5 & 5 & 5 & 5 & 5 & 5 & 5 & 5 & 5 & 5 \\
\hline 8 & 5 & 5 & 5 & 5 & 5 & 5 & 5 & 5 & 5 & 5 & 5 & 5 & 5 & 5 & 5 & 5 & 5 & 5 & 5 & 5 & 5 & 5 & 5 & 5 & 5 & 5 & 5 & 5 & 5 & 5 & 5 & 5 \\
\hline 9 & 5 & 5 & 5 & 5 & 5 & 5 & 5 & 5 & 5 & 5 & 5 & 5 & 5 & 5 & 5 & 5 & 5 & 5 & 5 & 5 & 5 & 5 & 5 & 5 & 5 & 5 & 5 & 5 & 5 & 5 & 5 & 5 \\
\hline 10 & 5 & 5 & 5 & 5 & 5 & 5 & 5 & 5 & 5 & 5 & 5 & 5 & 5 & 5 & 5 & 5 & 5 & 5 & 5 & 5 & 5 & 5 & 5 & 5 & 6 & 6 & 6 & 6 & \begin{tabular}{|l|l|}
5 \\
\end{tabular} & 5 & $\begin{array}{ll} \\
\end{array}$ & 6 \\
\hline 11 & 5 & 5 & 6 & \begin{tabular}{|l|}
6 \\
\end{tabular} & 5 & 5 & 5 & 6 & 5 & 5 & 6 & 6 & \begin{tabular}{|l|}
5 \\
\end{tabular} & \begin{tabular}{|l|}
5 \\
\end{tabular} & 6 & $\overline{6}$ & \begin{tabular}{|l|}
5 \\
\end{tabular} & \begin{tabular}{|l|}
5 \\
\end{tabular} & 6 & 6 & 5 & 5 & 6 & 6 & 6 & 6 & 6 & 6 & \begin{tabular}{|l|l|}
5 \\
\end{tabular} & $\overline{6}$ & \begin{tabular}{l|l|}
6 \\
\end{tabular} & 6 \\
\hline 12 & 5 & 5 & 6 & 6 & 5 & 5 & 6 & 6 & 5 & 5 & 6 & 6 & 5 & 5 & 6 & 6 & 5 & 5 & 6 & 6 & 5 & 5 & 6 & 6 & 6 & 6 & 6 & 7 & \begin{tabular}{|l|l|}
6 \\
\end{tabular} & 6 & 6 & 7 \\
\hline 13 & 5 & 5 & 6 & 6 & 5 & 5 & 6 & 6 & 5 & 5 & 6 & 6 & 5 & 6 & 6 & 6 & 5 & 6 & 6 & 6 & 5 & 5 & 6 & 6 & 6 & 6 & \begin{tabular}{|l|l|} 
\\
\end{tabular} & 7 & 6 & 6 & 6 & \\
\hline 14 & 5 & 6 & 6 & 6 & 5 & 5 & 5 & 5 & 5 & 5 & 5 & 5 & 5 & 6 & 6 & 6 & 5 & 5 & 5 & 6 & 5 & 6 & 6 & 6 & 6 & 6 & 6 & 6 & 5 & 5 & 5 & \\
\hline 15 & 6 & 6 & 5 & 5 & 5 & 5 & 5 & 5 & 5 & 5 & 5 & 5 & 5 & 6 & 5 & 6 & 5 & 5 & 5 & 5 & 6 & 6 & 6 & 6 & 6 & 6 & 6 & 6 & 5 & 5 & 5 & 5 \\
\hline 16 & 5 & 5 & 5 & 5 & 5 & 5 & 5 & 5 & 5 & 5 & 5 & 5 & 5 & 5 & 5 & 5 & 5 & 5 & 5 & 5 & 5 & 5 & 5 & 5 & 6 & 6 & 6 & 6 & 5 & 5 & 5 & 5 \\
\hline 17 & 5 & 5 & 5 & 5 & 5 & 5 & 5 & 5 & 5 & 5 & 5 & 5 & 5 & 5 & 5 & 5 & 5 & 5 & 5 & 5 & 5 & 5 & 5 & 5 & 5 & 5 & 5 & 5 & 5 & 5 & 5 & 5 \\
\hline 18 & 5 & 5 & 5 & 5 & 5 & 5 & 5 & 5 & 5 & 5 & 5 & 5 & 5 & 5 & 5 & 5 & 5 & 5 & 5 & 5 & 5 & 5 & 5 & 5 & & 5 & 5 & & \begin{tabular}{|l|l}
5 \\
\end{tabular} & 5 & & \\
\hline
\end{tabular}




\subsection{HORAS DE DESCONFORTO RESULTANTES}

Ao correlacionar os fluxos líquidos totais de estresse térmico com os níveis de conforto do ITS, para todas as cidades, obtém-se a tabela de níveis de conforto hora a hora (Figura 9), onde se vê mais claramente o efeito do albedo sobre as classes de conforto/desconforto térmico. Embora na maioria dos casos o total de horas de desconforto diárias seja o mesmo, nos cenários de verão com AA há um aumento visível de horas com nível 6 , "quente", para nível 7, "muito quente". Para o inverno, a variação do total de horas de desconforto e intensidade de desconforto não é tão pronunciada e o aumento de calor radiante devido ao albedo é muito ameno, provavelmente pouco eficiente na melhora do conforto de regiões frias, sendo, nesses casos, mais promissora a correta orientação do cânion. Investigações mais aprofundadas considerando períodos mais longos podem ser úteis para confirmar estes resultados preliminares. A princípio, é razoável dizer que, de maneira geral, para o território brasileiro, aumentar o albedo tende apenas a piorar as condições de conforto do pedestre no verão, sem alterações significativas no período de inverno.

\section{CONSIDERAÇÕES FINAIS}

Aumentar o valor do albedo das superfícies internas de cânions urbanos (pavimentação e paredes) mostrou pequena influência nas temperaturas do ar simuladas para a proporção de cânion estudada $(\mathrm{H} / \mathrm{W}=1)$. Uma vez que $\mathrm{H} / \mathrm{W}$ maiores resultam em maior sombreamento do cânion, pode-se concluir que aumentar o albedo de forma localizada, não contribui significativamente para a redução da temperatura do ar no interior do cânion, em grande parte do contexto climático brasileiro. Esta afirmação não contraria as recomendações de uso de materiais de alto albedo em coberturas, onde de fato apresentam benefícios, mas mostra que o interior de cânions urbanos possui uma dinâmica diferente.

A avaliação dos efeitos do albedo sobre o conforto térmico de pedestres sugere que de forma geral, para o território brasileiro aumentar o albedo representa prejuízo ao conforto térmico dos pedestres no verão, com pouco benefício de inverno. Este estudo foi feito para apenas um dia específico de céu claro de verão, e de inverno, e portanto, indica tendências. Uma análise de períodos mais longos, incluindo dias nublados e o período noturno pode resultar em quadro mais claro do balanço geral de conforto para o ano como um todo.

Os resultados mostraram que a orientação geográfica dos cânions tem um efeito relevante sobre os níveis de estresse térmico, e cânions orientados N-S resultaram em uma condição de menor estresse térmico para os pedestres, comparados a cânions L-O, devido ao efeito de sombreamento daqueles durante o início da manhã e final de tarde. 


\section{REFERÊNCIAS}

AGARWAL, M.; TANDON, A. Modeling of the urban heat island in the form of mesoscale wind and of its effect on air pollution dispersal. Applied Mathematical Modelling, v. 34, n. 9, p. $2520-2530$, set. 2010.

CAMARA, G. et al. SPRING: Integrating remote sensing and GIS by object-oriented data modelling. Compurters \& Graphics, v. 20, n. 3, p. 395-403, May-Jun 1996.

CARNIELO, E.; ZINZI, M. Optical and thermal characterisation of cool asphalts to mitigate urban temperatures and building cooling demand. Building and Environment, v. 60, p. 56-65, fev. 2013.

EMMANUEL, R.; FERNAND, H. J. S. Urban heat island in humid and arid climates: role of urban form and thermal properties in Colombo, Sri Lanka and Phoenix, USA. Climate Research, v. 34, n. 3, p. 241-251, set. 2007.

ERELL, E. et al. Effect of high-albedo materials on pedestrian heat stress in urban street canyons. Urban Climate, v. Available online, out. 2013. ISSN ISSN 2212-0955.

ERELL, E.; PEARLMUTTER, D.; WILLIAMSON, T. Urban Microclimate: Designing the spaces between buildings. 1. ed. Washington: Earthscan, 2011. ISBN 978-1-84407-467-9.

ERELL, E.; WILLIAMSON, T. Simulating air temperature in an urban street canyon in all weather conditions using measured data at a reference meteorological station. International Journal of Climatology, v. 26, n. 12, p. 1671-1694, out. 2006. ISSN ISSN 0899-8418.

GIVONI, B. Estimation of the effect of climate on human: development of a new thermal index. Technion-Israel Institute of Technology, Haifa, 1963.

GRIMMOND, C. S. B.; OKE, T. Turbulent heat fluxes in urban areas and a local-scale urban meteorological parameterization scheme (LUMPS). Journal of Applied Meteorology, v. 41, p. 792-810, 2002.

HOFFMANN, P.; SCHLUENZEN, K. H. Weather Pattern Classification to Represent the Urban Heat Island in Present and Future Climate. Journal of Applied Meteorology and Climatology, v. 52, n. 12, p. 2699-2714, dez. 2013.

JACOBSON, M. Z.; TEN HOEVE, J. E. Effects of Urban Surfaces and White Roofs on Global and Regional Climate. Journal of Climate, v. 25, n. 3, p. 1028-1044, fev. 2012.

MACDONALD, R. W. Modelling the mean velocity profile in the Urban Canopy Layer. Boundary-Layer Meteorology, Netherlands, v. 97, p. 25-45, out. 2000.

PEARLMUTTER, D.; BERLINER, P.; SHAVIV, E. Integrated modeling of pedestrian energy exchange and thermal comfort in urban street canyons. Building and Environment, v. 42, n. 6, p. 2396-2409, 2007.

RADHI, H.; SHARPLES, S. Quantifying the domestic electricity consumptions for airconditioning due to urban heat island in hot arid regions. Applied Energy, v. 112, p. 371-380, dez. 2013.

RODRIGUES DA SILVA, V. P. et al. Evaluating the urban climate of a typically tropical city of northeastern Brazil. Environmental Monitoring and Assesment, v. 161, n. 1-4, p. 45-59, fev. 2010.

RORIZ, M. Base de dados climáticos de 411 municípios brasileiros (ANTAC). Roriz

Bioclimática: conforto térmico e eficiência energética, 2012. Disponivel em:

$<$ http://www.roriz.eng.br/epw_9.html>. Acesso em: 12 abr. 2012.

YAGHOOBIAN, N.; KLEISSL, J. Effect of reflective pavements on building energy use.

Urban Climate, v. 2, p. 25-42, dez. 2012. 